\title{
Artificial Bubble Cloud Targets for Underwater Acoustic Remote Sensing
}

\author{
Paul A. Hwang \\ QUEST Integrated, Inc., Kent, Washington \\ Ronald A. Roy and Lawrence A. Crum \\ Applied Physics Laboratory, University of Washington, Seattle, Washington
}

(Manuscript received 15 February 1994, in final form 7 June 1995)

\begin{abstract}
This article describes a technique that uses polymer additives to suspend air bubbles to form stable artificial bubble clouds. The results presented include the range of polymer concentrations for an effective bubble suspension; the void fraction, bubble size distribution, and stability of the generated artificial bubble clouds; and the effects of polymer on the acoustic properties of the bubble clouds, especially the acoustic velocity. It is concluded that, for low-frequency applications (less than $1 \mathrm{kHz}$ ), polymer concentrations of less than $1 \%$ do not modify significantly the acoustic velocity of bubble clouds. The stability of the produced bubble clouds can be made to last from a few hours to days or even months depending on the polymer concentration and the bubble size. Because only a low polymer concentration and small void fraction (both less than $1 \%$ ) are needed to generate effective scattering, the density of the produced bubble cloud target and its response to hydrostatic pressure are similar to those of the ambient sea water; thus, adjustment to quasi-neutral buoyancy is not difficult. These results suggest that underwater acoustic targets and quasi-neutrally buoyant tracers that are small but have large acoustic cross sections can be produced with artificial bubble clouds suspended and stabilized by polymers.
\end{abstract}

\section{Introduction}

Wind, waves, and other environmental parameters produce ambient noise (Kerman 1984, 1988, 1993). Understanding the noise source and the capability to determine the source level are important in underwater acoustic data processing and signal analysis. Through the active study of sea-surface-generated sounds, it is revealed that bubbles within a cloud significantly modify the liquid compressibility, resulting in low-frequency acoustic emissions from a cloud of relatively small size. Recent scattering or experimental results show that a columnar cloud as small as $0.046 \mathrm{~m}$ in radius and $0.82 \mathrm{~m}$ long, with $0.003-0.025$ void fraction, can produce acoustic emissions at resonance frequencies from 680 to $250 \mathrm{~Hz}$ (Yoon et al. 1991). Field measurements of acoustic backscatter indicate that the target strength of a bubble cloud with a void fraction of 0.0025 and an equivalent radius of $0.42 \mathrm{~m}$ is at least $10 \mathrm{~dB}$ more than a reflective steel sphere $0.56 \mathrm{~m}$ in radius (Carey and Roy 1993; Roy et al. 1992). The characteristics of acoustic scattering of bubble clouds can be applied to the design of low-cost underwater targets for sonar calibration and the development of neutrally buoyant tracers for acoustic remote sensing of the ocean.

Corresponding author address: Dr. Paul Hwang, QUEST Integrated, Inc., 21414-68th Avenue South, Kent, WA 98032.
This article describes a technique to produce stable artificial bubble clouds. Section 2 presents a brief review of the acoustic scattering of bubble clouds. Section 3 discusses the design of a bubble-based acoustic target. Section 4 describes laboratory experiments conducted to determine the stability, physical properties, and acoustic properties of artificial bubble clouds. Section 5 concludes this article with a short summary.

\section{Acoustic scattering of bubble clouds}

The acoustical behavior of resonating bubbles in water and the significant modification of acoustic properties by a minute amount of air in the liquid have been thoroughly studied (Wood 1930; Minnaert 1933; Urick 1967). The scattering cross section of a resonant bubble is typically two to three orders of magnitude larger than its geometric cross section. The ratio of the two cross sections is determined by the damping coefficient, which has three components: radiation, thermal conductivity, and shear viscosity (Devin 1959). The resonance frequency $f_{R}$ of a single bubble is inversely proportional to the bubble size and increases with the square root of the ambient pressure. The solution is given in Minnaert (1933):

$$
f_{R} \approx \frac{1}{2 \pi a}\left(\frac{3 \gamma P_{A}}{\rho}\right)^{1 / 2},
$$


where $a$ is the bubble radius, $\gamma$ the polytropic exponent (taken to be 1.4 for an adiabatic condition), $\rho$ the water density, and $P_{A}$ the ambient pressure. The dependence of the water depth $z$ is introduced through the term $P_{A}$ (Urick 1967):

$$
f_{R} \approx \frac{3.25 \times 10^{6}}{a}(1+0.1 z)^{1 / 2}
$$

In this last expression, $f_{R}$ is in hertz, $a$ in micrometers, and $z$ in meters. A more accurate expression of resonance frequency takes into account the effects of surface tension and heat exchange between bubble and water. Equations (1) and (2), although very simple in appearance, are in error by less than $8 \%$ for air bubbles with radii greater than $2 \mu \mathrm{m}$ at sea level (Urick 1967; Clay and Medwin 1977). Based on these equations, the resonance frequencies of bubbles with radii between 10 and $1000 \mu \mathrm{m}$ range from 325 to $3.25 \mathrm{kHz}$ near a free surface and from 1078 to $10.78 \mathrm{kHz}$ at a $100-\mathrm{m}$ depth.

In the natural environment, especially near the water surface, higher concentrations of bubbles generally occur in the form of plumes or clouds, collective oscillations of the bubbles in a cloud have been shown to be capable of producing low-frequency acoustic emissions (Carey 1985, 1987; Prosperetti 1985, 1988a,b; Carey and Browning 1988; d'Agostino and Brennen 1988, 1989; Commander and Prosperetti 1989; Lu et al. 1990; Kumar and Brennen 1991, 1993; Yoon et al. 1991; Carey and Fitzgerald 1993; Crum et al. 1994; Loewen and Melville 1994). In addition to serving as an active noise source, the presence of a small fraction of air bubbles in water modifies significantly the compressibility of the liquid and the acoustic scattering from the resulting bubble-water mixture is enhanced considerably, especially at lower frequency (Wood 1930; Anderson 1950; Rschevkin 1963; Morse and Ingard 1968; Carey and Roy 1993; Roy et al. 1992; Carey et al. 1993). A detailed review of the theoretical development of acoustic scattering by bubble clouds is presented in Carey and Roy (1992). For an acoustically compact spherical bubble cloud, the fundamental resonance frequency $f_{0}$ and the relative backscatter intensity $I_{s} / I_{i}$ are given by (Carey and Roy 1993; Roy et al. 1992; Carey and Figtzgerald 1993)

$$
\begin{gathered}
f_{0} \approx \frac{1}{2 \pi R}\left(\frac{3 \gamma P_{0}}{\rho \chi}\right)^{1 / 2} \\
\frac{I_{s}}{I_{i}}=\frac{K^{6}}{9(k r)^{2}} \frac{\left[1-\left(x / x_{m}\right)\right]^{2}}{\left[1-\left(x / x_{m}\right) K^{2} / 3\right]^{2}+\left[\left(x / x_{m}\right) K^{3} / 3\right]^{2}},
\end{gathered}
$$

where $k$ is the acoustic wavenumber in liquid, $R$ the radius of the cloud, $\chi$ the void fraction, $r$ the distance between the acoustic receiver and the bubble cloud, $K$ $=k R, x=\rho c^{2}, x_{m}=\rho_{m} c_{m}^{2}, c$ is the speed of sound, and the subscript $m$ denotes the effective properties of the air-water mixture. (Note: Other approximate equations for the resonance frequency of bubble clouds have been given by different authors; some of these equations are presented in the appendix.) Comparing (3) with (1), it is clear that the resonance frequency of a bubble cloud is considerably lower than that of the constituent air bubbles. In the case of bubble doud scattering, the dominant parameters are the void fraction and the length scale of the cloud. The relative intensity at resonance corresponds to the condition that the first bracketed term in the denominator of (4) vanishes and can be expressed as

$$
\left(\frac{I_{s}}{I_{i}}\right)_{0}=\frac{\left[\left(x_{m} / x\right)-1\right]^{2}}{\left(k_{0} r\right)^{2}} \approx \frac{\left[\left(c_{m}^{2} / c^{2}\right)-1\right]^{2}}{\left(k_{0} r\right)^{2}},
$$

where the subscript 0 denotes the properties at cloud resonance. The scattered intensity at resonance is critically affected by the resonant acoustic wavelength and the ratio $c_{m} / c(5)$. The dependence on the cloud size is implicit through the resonance condition (3). Because the sound velocity in a liquid with air bubbles is much lower (approximately $100 \mathrm{~m} \mathrm{~s}^{-1}$ with a 0.01 void fraction) than that of the bubble-free fluid (on the order of $1500 \mathrm{~m} \mathrm{~s}^{-1}$ in water), the target strength [in decibels, defined as $10 \log \left(I_{s} / I_{i}\right)$ at $r=1 \mathrm{~m}$ ] of a bubble cloud at resonance is on the order of $0 \mathrm{~dB}$, and the bubble cloud resonance frequency is on the order of $100 \mathrm{~Hz}$ (see next section). In particular, if the void fraction is greater than 0.01, then $c_{m} \ll c($ Wood 1930) and the last expression in (5) reduces to $k_{0}^{-2}$, suggesting, that the target strength at resonance increases as the collective resonance frequency decreases.

Successful experimental verifications of the collective oscillations of bubbles have been carried out in the laboratory (Kolaini et al. 1991; Yoon et al. 1991). The bubbles were generated by hypodermic needles arranged in concentric circles. The geometry of the bubble clouds is cylindrical. The height of the bubble column is (nominally) $0.82 \mathrm{~m}$. With a bubble cloud 0.046 $\mathrm{m}$ in radius, the measured resonance frequency outside the bubble column ranges from $680 \mathrm{~Hz}$ with a 0.003 void fraction to $250 \mathrm{~Hz}$ with a 0.025 void fraction. These frequencies are substantially lower than the resonance frequency of the constituent bubbles, which had a narrow size distribution with a mean radius of $1.6 \mathrm{~mm}$. The agreement between the measured and predicted resonance frequencies is excellent.

Acoustic scattering from bubble clouds was measured in a field experiment performed at Lake Seneca (Carey and Roy 1993; Roy èt al. 1992). The bubble clouds were produced at a depth of $91.4 \mathrm{~m}$ by bursts of pressurized air through hypodermic needles arranged in concentric circles. The measured data show evidence of resonance scattering peaks at $0.45,1.3,3.0,6.5$, and possibly $11 \mathrm{kHz}$ from a bubble cloud with an elongated teardrop geometry (diameter $\approx 0.5 \mathrm{~m}$, length $\approx 1.4$ 
$\mathrm{m}$, void fraction $\approx 0.0025$ ). The lowest resonance frequency is in good agreement with the theoretical prediction, assuming an equivalent spherical cloud of $R$ $=0.42 \mathrm{~m}$ and an isothermal condition $(\gamma=1$; see the discussion in Carey and Browning 1988). The target strength at these resonance peaks ranges from -4 to 1 dB. For comparison, the target strength of a reflecting steel sphere $1.12 \mathrm{~m}$ in diameter is measured to be -12.3 $\mathrm{dB}$ at $5 \mathrm{kHz}$ with an identical setup. These results strongly suggest that bubble clouds of a relatively small size can serve as very efficient undersea acoustic targets.

\section{Design of bubble cloud acoustic targets}

\section{a. Conceptual discussion}

To produce consistent undersea bubble-based targets, the geometry and void fraction of the bubble cloud need to remain stable. The basic approach is to design a means to contain the bubbles in an acoustically transparent vessel or medium. The key parameters that determine the resonance frequency of a bubble cloud target are the void fraction $\chi$, the length scale of the bubble cloud $R$, and the depth $z$ underwater (Hwang et al. 1995). The resonance frequency of bubble cloud targets computed using (3) for $\chi=0.01$ to $0.15 ; R$ $=0.1$ to $1.0 \mathrm{~m}$; and $z=0$ to $100 \mathrm{~m}$ are presented in Fig. 1. These results show that relatively small targets can produce low-frequency acoustic resonance, especially in the upper layer of the ocean column. However, as noted in Carey and Roy (1993), the presence of the air-water interface introduces a mirror effect. The dipole characteristic reduces the target strength of bubble clouds near the air-water interface (Schindall et al. 1992; Crum et al. 1994).

The relative backscatter intensity $I_{s} / I_{i}$ at resonance predicted from (5) is illustrated in Fig. 2a, showing the $k_{0}^{-2}$ dependence. The results in terms of target strength are presented in Fig. 2b. As a comparison, the backscatter target strength as a function of frequency for a compressible sphere $(0.25 \mathrm{~m}$ in radius, 0.01 void fraction) in free space (Fig. 2c) is calculated using a full partial-wave solution (Anderson 1950; Morse and Ingard 1968; Roy et al. 1994). The frequency and target strength of the fundamental mode, $97 \mathrm{~Hz}$ and $8 \mathrm{~dB}$, respectively, are in excellent agreement with the results calculated from (3) and (5).

Because volume and weight increase as the third power of the radius for a spherical object, it may become an important consideration in the design of large targets. For example, the volumes of the five spheres (radii $0.1,0.15,0.2,0.25$, and $1.0 \mathrm{~m}$ ) in Fig. 1 are 4.2, $14.1,33.5,65.4$, and $4189 \mathrm{~L}$, respectively. The rapid increase of target weight with size becomes critical in field deployment. An ideal design would permit the option of bubble cloud generation in situ and thus alleviate the weight problems of target transportation and deployment.

\section{b. Bubble suspension and stabilization by polymer additives}

One way to "suspend and stabilize" bubbles in water is by polymer additives, as widely used in many industries to produce uniform suspensions of solid or gas in liquids. The applications include drilling and oil recovery (Gray and Darley 1981) and the processing of food, pharmaceuticals, abrasives, coatings, and ceramics (Kelco 1988). The polymer of special interest to this project is xanthan gum (known also as XC polymer), a microbial polysaccharide produced by fermentation. The xanthan gum production method was developed at the Northern Regional Research Center, Agricultural Research Service, USDA, Peoria, Illinois, in 1961. Kelco commercialized xanthan gum in 1964, and the product has been approved in the United States for general use in foods since 1969. Xanthan gum derives its superior suspension-stabilization property from viscosity buildup in water and salt water-a more than two-order-of-magnitude increase from $0.1 \%$ to $1 \%$ gum concentration. Xanthan gum solutions also display exceptional shear-thinning properties (Kelco 1988).

Viscosity buildup and shear thinning are ideal for the production of bubble cloud targets; aeration can be introduced from bubblers or orifices into a xanthan gum solution, and the aerated mixture can be conveniently pumped directly into the ocean or stored in target containers. For storage, the bubble cloud mixture can be pumped into a pressurized vessel, the pressure of which is set to equalize the ambient pressure at the target deployment depth. Or excessive air can be introduced in the polymer solution to compensate for the expected void fraction reduction due to the hydrostatic pressure at the deployment depth. Once produced, the size and number of bubbles can be maintained for an extended period of time (see next section). The bubble cloud structure will remain the same (in the case of pressurized storage) or can be calculated (in the case of unpressurized storage) upon release to the desired depth.

Because the viscosity of a xanthan gum mixture is greatly reduced at a high shear, it offers a practical method for in situ production and simple deployment of large bubble cloud targets in the field. Figure 3 presents a conceptual sketch showing the on-site operation. Note the flexibility of varying the relevant parameters, such as void fraction, cloud size, and depth of release. For very large bubble cloud targets, this is probably the most practical approach. The above design can be applied to any geometry such as oval or conic shape to simulate breaking-wave-induced bubble plumes at different stages of the breaking process. For a simple geometry, analytical solutions can be derived to predict the acoustic scattering properties of the bubble clouds. For a more complicated geometry, numerical procedures can be developed to provide quantitative design parameters. Alternatively, the acoustic properties of the 

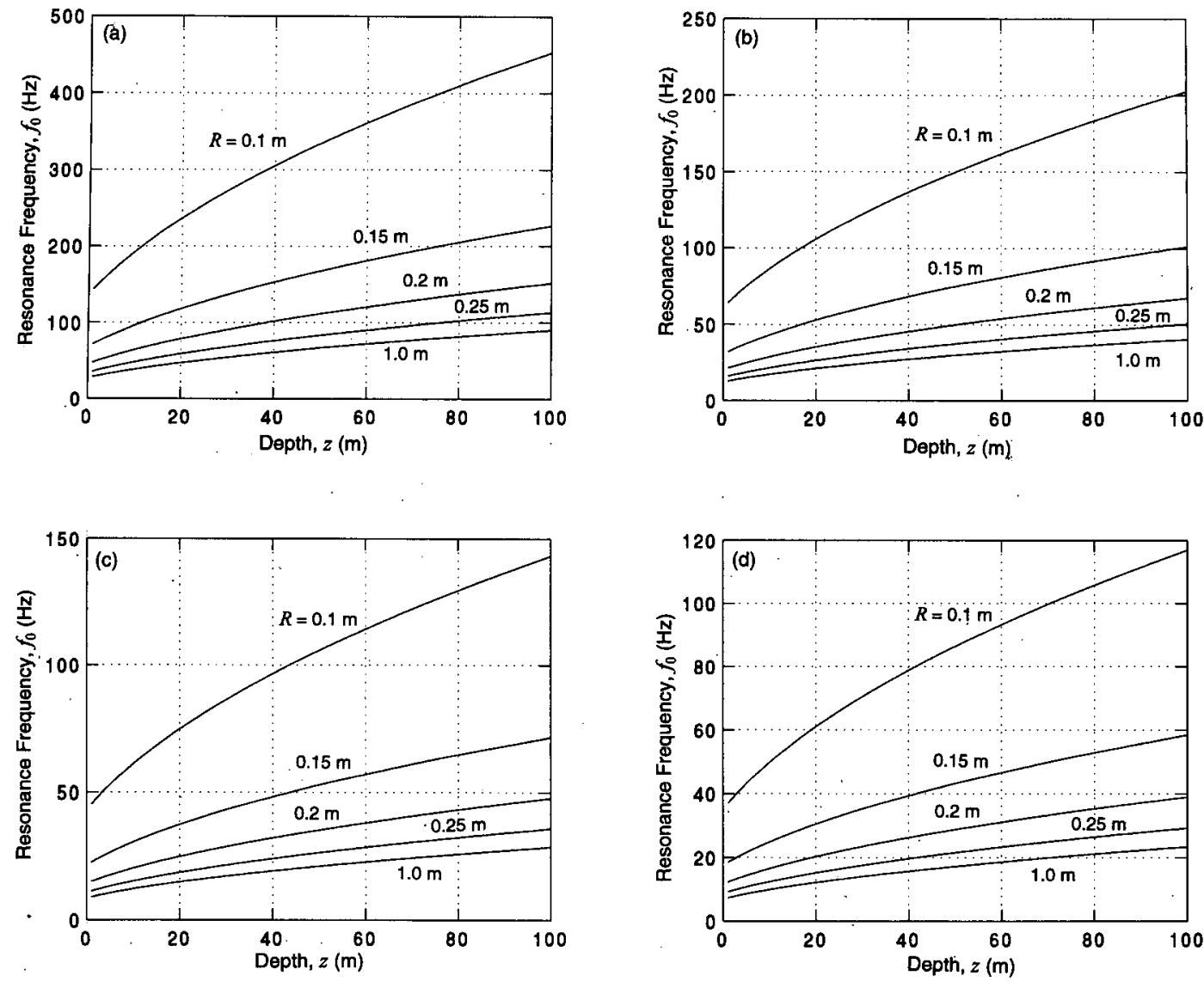

FIG. 1. Bubble cloud resonance frequency as a function of water depth: $R=0.1,0.15,0.2,0.25$, and $1.0 \mathrm{~m}$ (increasing downward); (a) $\chi=0.01$; (b) $\chi=0.05$; (c) $\chi=0.10$; and (d) $\chi=0.15$.

artificial bubble clouds with complicated geometry can be characterized by laboratory and field measurements.

\section{c. Deployment strategy}

The techniques to deploy a bubble cloud target depend on many factors, including the local water depth, the target depth, and whether pressurized storage is used. The discussion below is only qualitative.

When tethering is used, such as in the application of sonar calibration, the bubble cloud targets can be attached on lines and lowered to the desired depth. They can also be anchored at the bottom in shallow water or suspended from buoys in deep water. To maintain a special orientation of a nonspherical target, weights can be added at the bottom of the apparatus and floats at the top. By properly adjusting the locations of weight and float attächments, different geometric projections with respect to the sonar devices can be arranged. Once the desired target depth is reached, the emulsified bubble clouds are allowed to adjust to the local pressure and temperature. Since the bubble size at a given time can be predicted or calibrated, the quantitative relationship of sonar measurements and the bubble cloud characteristics can be analyzed. The void fraction can be determined directly from the number of bubbles introduced at the time of target production and knowledge of the bubble size at the deployed depth.

Untethered applications of the bubble cloud targets, such as for tracing ocean currents or pollutant plumes, can be deployed from a surface ship or subsurface vehicle. It is emphasized that the density of the artificial bubble-based targets typically differs from the ambient water by less than $1 \%$. Superb flow-following performance is expected without any elaborate mecharisms for density adjustment.

\section{Laboratory experiments}

The analysis presented earlier demonstrates convincingly that artificial bubble clouds can be produced inexpensively, with promising applications in the design of acoustic targets for underwater acoustics research and the design of neutrally buoyant tracers for acoustic remote sensing of the ocean. It remains to be proven that 1) bubbles within a suitable size range can be stabilized in a polymer mixture; and 2) the resulting 

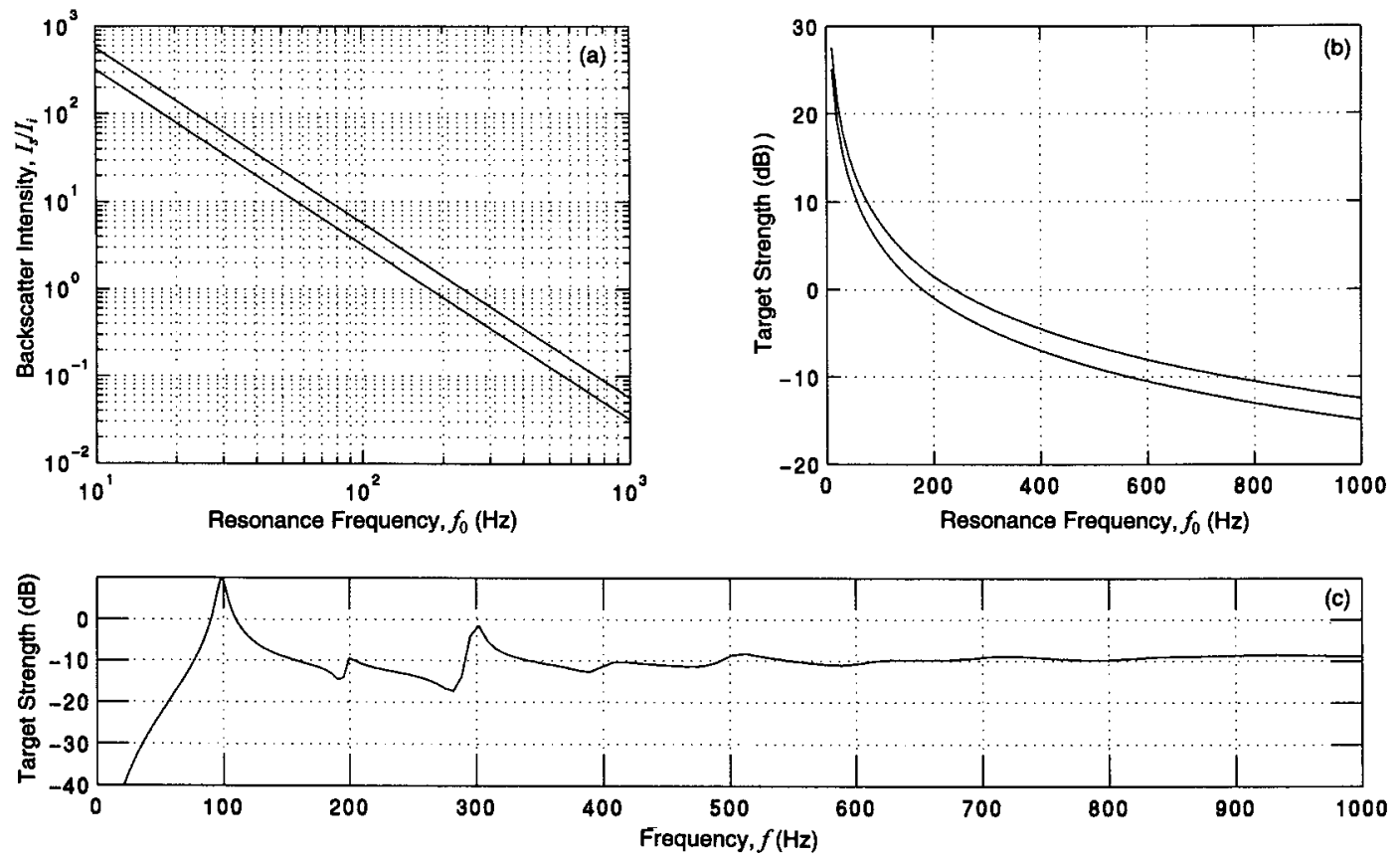

FIG. 2. (a) Backscatter intensity and (b) target strength at resonance frequency of bubble cloud. Upper curves: $c_{m} / c$ $=0.05$; lower curves: $c_{m} / c=0.5$. (c) Target strength of a compressible sphere $(R=0.25 \mathrm{~m}, \chi=0.01)$ computed using a full partial-wave solution (Anderson 1950; Morse and Ingard 1968; Roy et al. 1994).

bubble cloud will be stable for a reasonable period, from a few hours to a few months depending on applications. It is also important to determine the effects of polymer addition on the acoustic properties of the artificial bubble clouds. A series of laboratory experiments was conducted to answer these questions. The

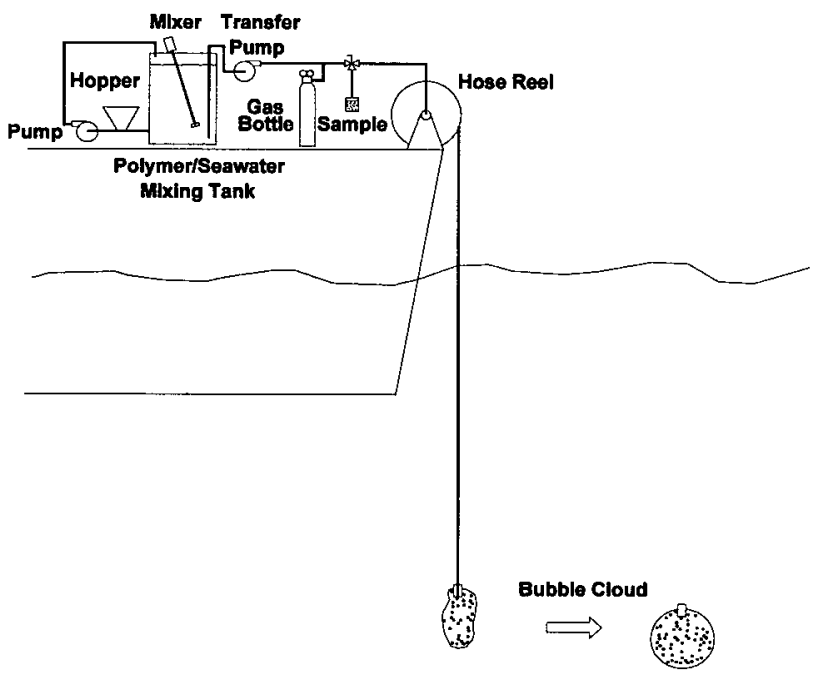

FlG. 3. A sketch showing the production and deployment of bubble cloud targets on a ship. The air bubbles are suspended and stabilized in the cloud by polymer additives. Large targets can be produced in situ at the desired deployment depth. experimental procedures and the results are described in this section.

\section{a. Bubble suspension and polymer concentration}

\section{1) EXPERIMENTAL PROCEDURE}

The first task was to understand the relationship between the size of the suspended bubbles and the polymer concentration. Polymer mixtures with concentration ranges from $0.05 \%$ to $1.0 \%$ were prepared in test tubes. Air bubbles were then introduced into the mixture by shaking. The evolution of bubbles in the mixture was recorded both by visual inspection and photographs. The experiments were repeated three times over a period of three months. The tests, though simple, quickly established a narrow range of polymer concentrations for more detailed study.

\section{2) Results}

From these simple tests, it was found that polymer mixtures with less than $0.2 \%$ concentration could suspend only very small bubbles that disappeared within an hour, which would be of little use in field applications where preparation and equipment setup take time. To suspend bubbles for a meaningful period (nominally more than $1 \mathrm{~h}$ as a realistic time for field deployment), the polymer concentration needed to be at least $0.4 \%$. Table 1 summarizes the results from the tests with polymer concentrations ranging from $0.2 \%$ 
TABLE 1. Visual observations of the capability of polymer mixtures to suspend and stabilize bubbles as a function of polymer concentration.

\begin{tabular}{|c|c|c|c|c|c|}
\hline Concentration (\%) & 0.2 & 0.4 & 0.6 & 0.8 & 1.0 \\
\hline $\begin{array}{l}\text { Max. bubble diameter } \\
\text { Suspending duration }\end{array}$ & $\begin{array}{l}\sim 0.1 \mathrm{~mm} \\
\sim 1 \mathrm{~h}\end{array}$ & $\begin{array}{l}\sim 1 \mathrm{~mm} \\
\sim 1 \text { day }\end{array}$ & $\begin{array}{l}\sim 1.5 \mathrm{~mm} \\
\text { days }\end{array}$ & $\begin{array}{l}\sim 3 \mathrm{~mm} \\
\text { weeks }\end{array}$ & $\begin{array}{l}-3 \mathrm{~mm} \\
\text { months }\end{array}$ \\
\hline
\end{tabular}

to $1.0 \%$. It is noted that, at the high end of the polymer concentration, the trapped bubbles stayed in the mixture for a very long period of time-all the large bubbles in the millimeter size range were still trapped inside the $1 \%$ mixtures prepared on the first and thirtieth days at the time of the last observation at the end of the three-month period.

\section{b. Void fraction and size distribution}

\section{1) EXPERIMENTAL PROCEDURE}

The second step was to obtain the physical characteristics of the polymer mixtures. Two particular properties were of most interest to this study: void fraction and size distribution. These properties obviously were functions of the generation process. At this stage, we had not developed a reliable technique to produce monodisperse bubble clouds in the polymer solution. Instead, a few simple methods of bubble injection into the polymer mixtures were tried. These included mechanical mixing, Venturi suctioning, submerged bubbling, and jet splashing. The last two methods were used for most of the experiments reported herein. Typically, a void fraction of approximately 0.03 can be achieved within about $5 \mathrm{~min}$. Three polymer concentrations (by weight) were used in the experiment: $0.4 \%$, $0.6 \%$, and $0.8 \%$. The procedures to derive the void fraction and size distribution are described below.

\section{(i) Void fraction}

Two methods were used to determine the density of the mixture, which was then used to calculate the void fraction.

1) Measuring the weight of a known volume of mixture: Four flasks with calibrated volumes between 106 and $109 \mathrm{~cm}^{3}$ were used for this measurement. With a measuring accuracy of $0.05 \mathrm{~g}$ (a task that can be achieved with a simple balance), the determination of the mixture density was better than 0.0005 (absolute uncertainty), which was sufficient for the present task aimed at producing high-void-fraction bubble targets. (Note: If the aim is to simulate the natural environment, where the ambient void fraction is on the order of $10^{-5}$, the required accuracy would be much more stringent.)

2) Measuring the submerged weight of a balloon filled with polymer mixture: In this experiment, the bubble mixture was injected into small balloons to ap- proximately $0.1 \mathrm{~m}$ in diameter. In preparing the balloons, it is important to "squeeze" out the air pocket so that the void fraction is contributed only by air bubbles suspended in the polymer mixture. Typically four balloon samples were used. The density of the mixture in the balloons was measured using a simple procedure that included (a) measuring the weight $W$ of the balloon in air and (b) attaching a sinker of known submerged weight and measuring the submerged total weight of the balloon and sinker. These measurements provided information on the buoyancy $B$ of the balloon. The density of the mixture $\rho_{m}$ in the balloon was then determined by the equation

$$
\frac{\rho_{m}}{\rho_{w}}=\frac{W}{W+B} .
$$

Note that in (6), $W+B=\rho_{w} V$ is the product of the water density, $\rho_{w}$, and the volume of the mixture, $V$. This is derived from $B=\left(\rho_{w}-\rho_{m}\right) V$ and $W=\rho_{m} V$; the water density was assumed to be $1000 \mathrm{~kg} \mathrm{~m}^{-3}$. The last assumption may introduce a relative error of approximately $0.2 \%$ in the temperature range encountered in the experiment.

The results from the two methods were found to be compatible. Using either method, the sample density or the submerged weight of the balloon was monitored at regular intervals and the density variation of the polymer as a function of time could be determined.

\section{(ii) Size distribution}

The size distribution of bubbles was derived from photographic images of a small sample $\left(15 \mathrm{~cm}^{3}\right)$ in a petri dish. The images were recorded by a two-dimensional CCD camera $(512 \times 480$ pixels $)$. Test runs indicated that the resolving capability was about two to three pixels. To cover the (radius) size range from 20 to $1000 \mu \mathrm{m}$, two different fields of view (FOVs) were used, nominally $5 \mathrm{~mm} \times 7 \mathrm{~mm}$ and $20 \mathrm{~mm} \times 28 \mathrm{~mm}$. The depth of the sample in the petri dish was $3.4 \mathrm{~mm}$, based on calculations. The relatively large thickness was needed because bubbles $2-3 \mathrm{~mm}$ in diametes: were not uncommon, as will be shown later. A commercial image processing software (ImagePro Plus) was used for sizing and counting to derive the size distribution of bubbles in the polymer mixture.

\section{2) Results}

The results from two experiments are reported below: a long-term monitoring program $(0.8 \%$ polymer 
concentration, $95 \mathrm{~h}$ ) and a short-term observation of bubble stability $(0.4 \%, 0.6 \%$, and $0.8 \%$ polymer concentrations, $2-4 \mathrm{~h}$ ).

\section{(i) Long-term monitoring}

The polymer mixture was prepared with $6 \mathrm{~L}$ of tap water and $0.048 \mathrm{~kg}$ of Kelzan S, resulting in a $0.8 \%$ solution. The polymer was stirred gradually into the water and pumped to enhance mixing. During this mixing process, a small amount of air was introduced into the mixture. The density and bubble size distribution were recorded (as described in the last section) before air injection to serve as a reference. The mixture density before aeration was 0.9958 (Table 2). The size of the bubbles introduced through mixing was less than $300 \mu \mathrm{m}$ (Fig. 4). Analysis of the photographic images indicates that the bubble population follows a power-law distribution, and the slope of the size distribution is -5 (Fig. 5).

After the reference measurements were taken, air bubbles were introduced through submerged bubblers by a pressurized air supply with a pressure drop of 20000 $\mathrm{Pa}$ for $5 \mathrm{~min}$. The pump was turned on during the bubbling process and kept on for a period of 5-10 min after aeration to mix the fluid. Next, the density of the mixture was measured and photographic images taken. The results indicate a void fraction of $0.039(=0.043-0.0042)$ was introduced into the polymer mixture during the 5min bubbling process (Table 2). Bubbles as large as 4 $\mathrm{mm}$ in diameter were trapped in the polymer solution (Figs. 4 and 5). The spectral slope is noticeably flattened to -3.5 . As mentioned earlier, in order to cover the bubble size over the range of $20-1000-\mu \mathrm{m}$ radius, at least two different amplifications are needed. For each size spectrum shown in Figs. 4 and 5 , the number of bubbles is cumulated over a fixed size band $(25 \mu \mathrm{m})$ from five different photographic images.

Subsequent measurements of mixture density and bubble images were taken at $16.5,24.5,41,65,72$, and $95 \mathrm{~h}$

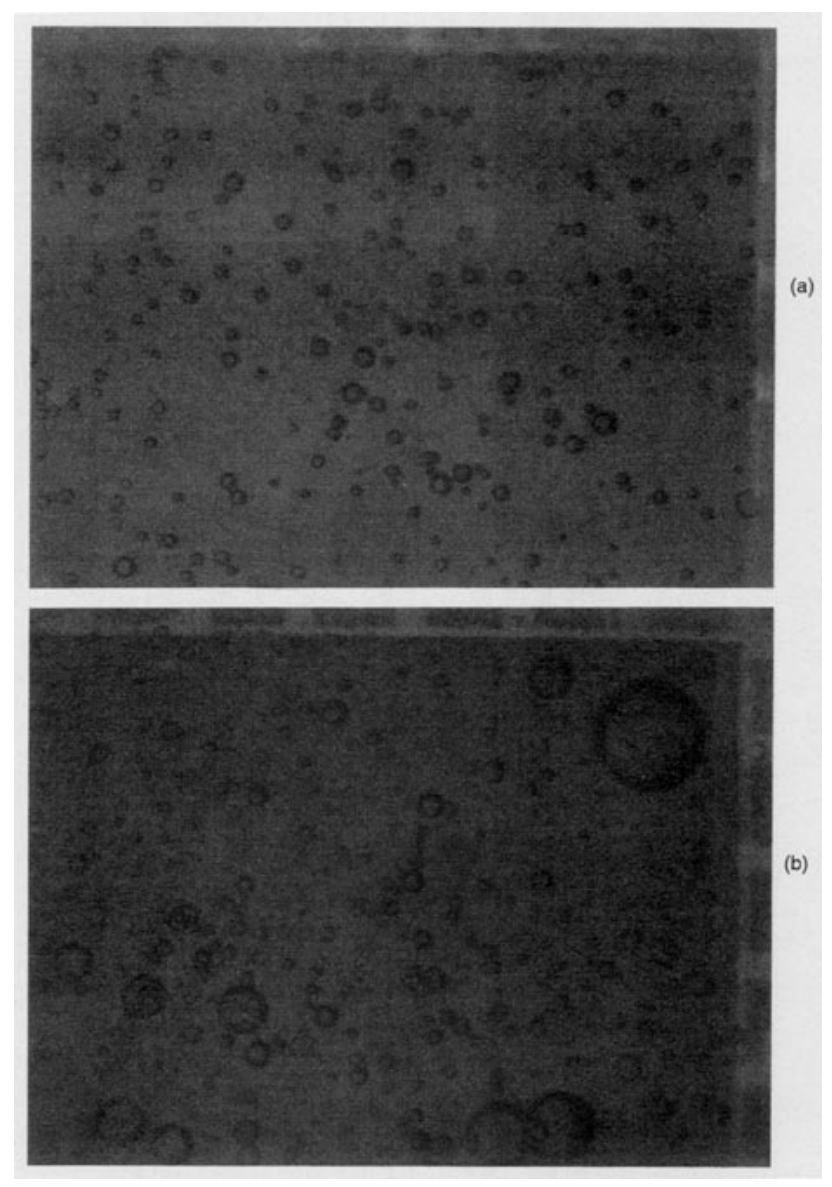

FIG. 4. Photographic images of bubbles in a $0.8 \%$ polymer solution. (a) After pump mixing and before air injection; (b) after $5 \mathrm{~min}$ of bubble injection from two submerged bubblers at $20000-\mathrm{Pa}$ air pressure. The spacing between tick marks on the edges of the photograph is $1 \mathrm{~mm}$.

after bubble injection. From the density data (Table 2), it was found that the void fraction decreased in the first $24 \mathrm{~h}$ or so from 0.043 to 0.029 . Interestingly, an increase

TABLE 2. Density and void fraction of a bubble mixture in a $0.8 \%$ polymer solution.

\begin{tabular}{|c|c|c|c|c|c|c|}
\hline Time $^{\mathrm{a}}$ & $T_{\text {air }}\left({ }^{\circ} \mathrm{C}\right)$ & $\begin{array}{l}T_{\text {fluid }} \\
\left({ }^{\circ} \mathrm{C}\right)\end{array}$ & $\underset{(\mathrm{mm} \mathrm{Hg})}{P_{\text {atm }}}$ & $\begin{array}{c}\text { Humidity } \\
(\%)\end{array}$ & $\begin{array}{c}\rho_{m}^{b} \\
\left(\mathrm{~g} \mathrm{~cm}^{-3}\right)\end{array}$ & $\begin{array}{c}\text { Void } \\
\text { fraction }\end{array}$ \\
\hline$<5 \mathrm{~min}$ & 22.5 & & & & ${ }_{2} 0.9958$ & 0.0042 \\
\hline $12 \mathrm{~min}$ & & & & & ${ }_{3} 0.9570$ & 0.0430 \\
\hline $16.5 \mathrm{~h}$ & & & & & ${ }_{1} 0.9655$ & 0.0345 \\
\hline $24.5 \mathrm{~h}$ & 21.8 & 21.7 & & & 0.9711 & 0.0289 \\
\hline $41 \mathrm{~h}$ & & & & & 0.9654 & 0.0346 \\
\hline $65 \mathrm{~h}$ & 21.0 & 21.0 & 744 & 66.8 & 0.8684 & $0.1316^{\mathrm{c}}$ \\
\hline $72 \mathrm{~h}$ & 21.9 & 21.2 & 746 & 64.0 & 0.9081 & 0.0919 \\
\hline $95 \mathrm{~h}$ & 21.5 & & 750 & 63.5 & 0.9377 & 0.0623 \\
\hline
\end{tabular}

${ }^{a} t=0$ at the time when bubble injection (for $5 \mathrm{~min}$ ) stopped.

${ }^{b}$ Average of four samples; otherwise the number of samples is denoted as the left subscript.

${ }^{c}$ No preservatives were added in the polymer solution. After $65 \mathrm{~h}$, the size spectrum changed significantly, showing abundant bubbles in the size range $400 \pm 200 \mu \mathrm{m}$. The void fraction was also increased by a factor of nearly 4 (from 0.035 to 0.132 ) within $24 \mathrm{~h}$. The emitted strong odor suggested that fermentation contributed to the increase in the bubble population and the narrowing of the bubble size distribution. 

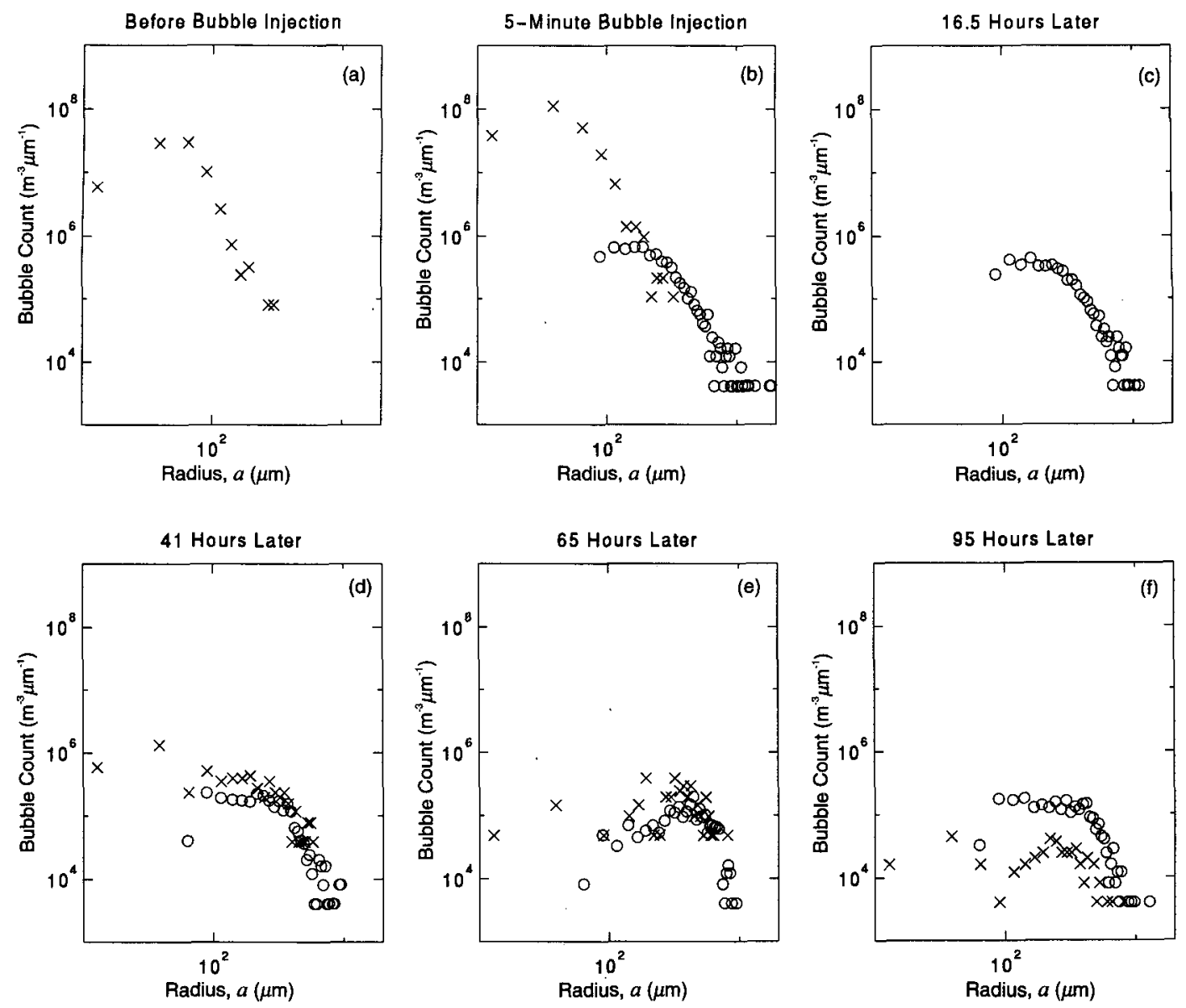

FIG. 5. Bubble size spectra in a $0.8 \%$ polymer solution at different times (marked on top of each panel) before and after bubble injection from two submerged frits at $20000-\mathrm{Pa}$ air pressure. The crosses and circles denote the results from two different magnifications (FOVs).

in the void fraction followed, reaching a level of 0.132 at $65 \mathrm{~h}$. The size spectrum at this stage was also significantly different from earlier samples, showing a distinctively narrower size distribution with a large number of bubbles in the size range $a \approx 400 \pm 200 \mu \mathrm{m}$. The cause of this drastic change was later determined to be due to the fermentation process (a strong odor provided the hint). Passing this peak of fermentation, the void fraction again reduced gradually to 0.092 and 0.063 on the third and fourth day, respectively. The size spectra at $t=16.5,41,65$, and 95 $\mathrm{h}$ are also presented in Fig. 5. All the resulting spectra are combined in Fig. 6. It was found that the population of large bubbles remained relatively invariant with time and, in the case of active fermentation, increased, while the smaller bubbles disappeared within the first two days. This variation in the bubble size spectra suggests that the dissolution of small bubbles in the mixture is the primary mechanism for the change of bubble characteristics. Reports of the dissolution of small bubbles ( $<20$ or $30 \mu \mathrm{m}$ ) have been noted in other studies of air bubbles in water by Blanchard and Syzdek (1972), who state that in laboratory experiments such small bubbles rise from the gen- erating capillary tube for a few centimeters and "disappear into nothingness" (see also Blanchard 1983). Ward et al. (1982) study the stability of bubbles in a closed volume of liquid-gas solution and predict that, "depending on the amount of gas present in the volume, there may be no equilibrium state for the bubble or bubbles, one equilibrium size, or two possible equilibrium sizes. In the latter case, it is also predicted that the equilibrium state corresponding to the larger bubble size is a stable equilibrium state." Our observations on the bubble size evolution conform to the prediction that the larger bubbles are at a stable equilibrium state.

\section{(ii) Short-term observation}

The results on the temporal variation of the void fraction for the short-term observations of three mixtures $(0.4 \%, 0.6 \%$, and $0.8 \%$ polymer concentrations ) are summarized in Fig. 7. These results were obtained from balloon measurements as described in the last section. On the left column of the figure, the absolute void fractions of four individual balloon samples are 

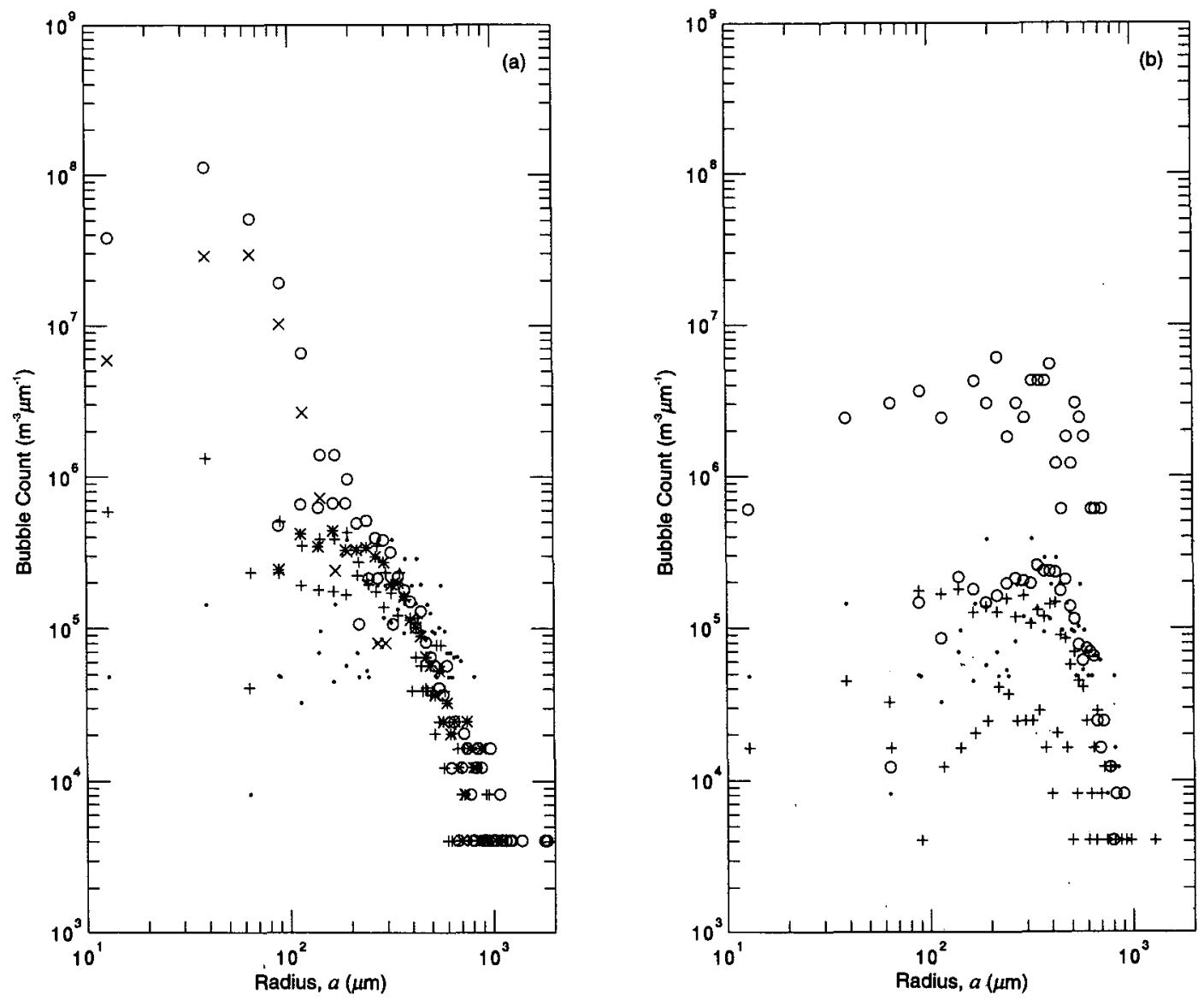

FIG. 6. Combined bubble size spectra in a $0.8 \%$ polymer solution at different times after bubble injection. Symbols: (a) cross-before bubble injection, circle-12 $\mathrm{min}$ after 5 -min bubble injection, asterisk-after $16.5 \mathrm{~h}$, plus-after 41 h, dot-after $65 \mathrm{~h}$; (b) dot-after $65 \mathrm{~h}$, circle-after $72 \mathrm{~h}$, plus-after $95 \mathrm{~h}$.

plotted as a function of elapsed time after bubble injection. Note that the sample-to-sample variation of the void fraction is rather large, indicating that in order to produce a homogeneous artificial bubble cloud more mixing is needed with the present method of air injection. Regardless of this drawback, the variation of the void fraction of each balloon is quite small over the observation period, ranging from 2 to $4.5 \mathrm{~h}$. The normalized void fraction is plotted in the right column, showing that in most of the cases the relative change of the void fraction is less than 5\% for a period of $2 \mathrm{~h}$. Also interesting in this figure is that the variation of void fraction can either increase (!) or decrease with time, as shown in the bottom panels $(0.8 \%$ polymer concentration ). This appears to be due to the temperature condition at the time of bubble cloud preparation. However, our data on this parameter are limited at this stage. Further investigation is in progress.

\section{c. Acoustic properties of bubble clouds in a polymer mixture}

The ultimate question to be answered in developing the artificial bubble cloud targets or tracers using polymer solutions is their acoustic properties. Because the acoustic velocity in the mixture is a critical parameter determining the acoustic scattering of bubble clouds, see (3) and (4), it is pertinent to measure the acoustic velocity in the artificial cloud. Also, because the primary modification of the polymer addition is an increase of viscosity by two to three orders of magnitude, it is a concern whether the acoustic absorption is significantly increased. Both the acoustic velocity and the absorption coefficient in the polymer mixture can be derived from the resonant tube experiments (Carsten and Foldy 1947; Silberman 1957; Coste and Laroche 1993).

\section{1) EXPERIMENTAL PROCEDURE}

The acoustic resonator is a vertical pipe made of Plexiglas $1.3 \mathrm{~m}$ long with a circular cross section $0.076 \mathrm{~m}$ in diameter. Monotonic excitation is generated at the bottom from a balloon membrane connected to a shaker through a piston. The shaft of the piston passes through a large rubber stopper to isolate direct excitation from the shaker to the tube wall. A hydrophone with a sensor head $0.01 \mathrm{~m}$ in diameter traverses the 

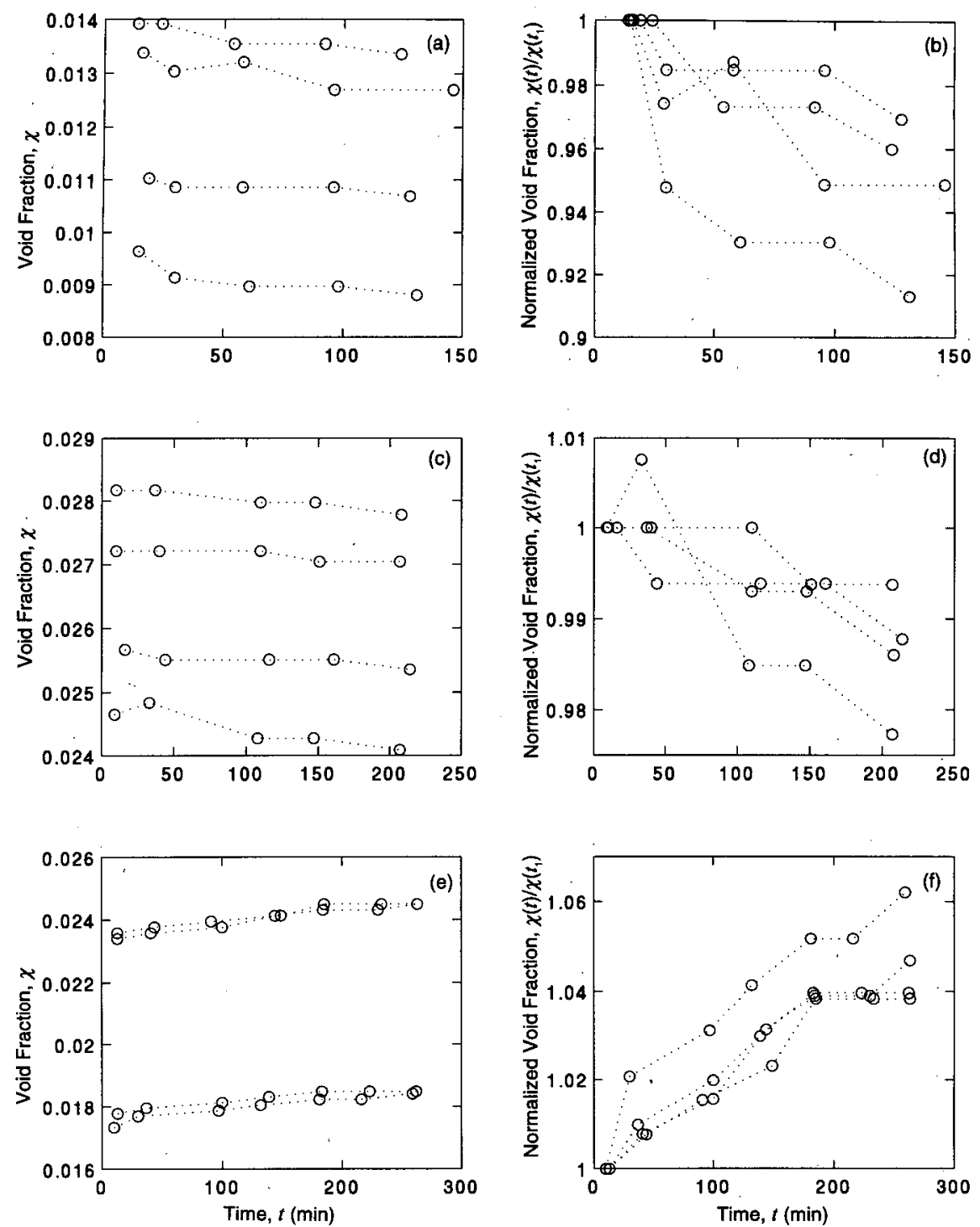

FIG. 7. Stability of artificial bubble mixtures as indicated by the temporal variation of void fraction. The polymer concentration is $(a, b) 0.4 \%$; $(c, d) 0.6 \%$; and $(e, f) 0.8 \%$. The left column plots the absolute void fraction of four balloon samples for each polymer concentration; the right column plots the normalized void fraction.

full length of the pipe to map out the standing wave pattern. In the earlier experimental runs, the reading was taken every $0.05 \mathrm{~m}$. Later, the spacing was reduced to $0.025 \mathrm{~m}$. Two sequences of measurements, one with the hydrophone traversing upward and the second with it traversing downward, were taken to check the repeatability of the results. At each position, 3.2 or $8.0 \mathrm{~s}$ of hydrophone output was recorded. The positions of nodes and antinodes can be identified from the distribution of acoustic pressure. The phase velocity was derived from the wavelength and the applied acoustic frequency (Silberman 1957). The attenuation coefficient $\alpha$ can be estimated from the ratio of acoustic pressure at the node and antinode, $P_{N} / P_{A}$, as also described in Silberman (1957):

$$
\alpha=\frac{4 f}{c} \tanh ^{-1} \frac{P_{N}}{P_{A}} .
$$

Two series of experiments were conducted:

(a) Acoustic measurements in bubbly water: In this series, air bubbles were injected from the hypodermic needles inserted along the circumference of the resonant tube at an elevation $0.03 \mathrm{~m}$ from the bottom. By adjusting the pressure of the air supply, the void fraction in the water could be controlled. The void fraction was determined from the water elevations before and after air injection. For a $1-\mathrm{m}$ nominal water height and a $0.5-\mathrm{mm}$ reading accuracy, the uncertainty of the void fraction derived from this method was \pm 0.0005 . The 

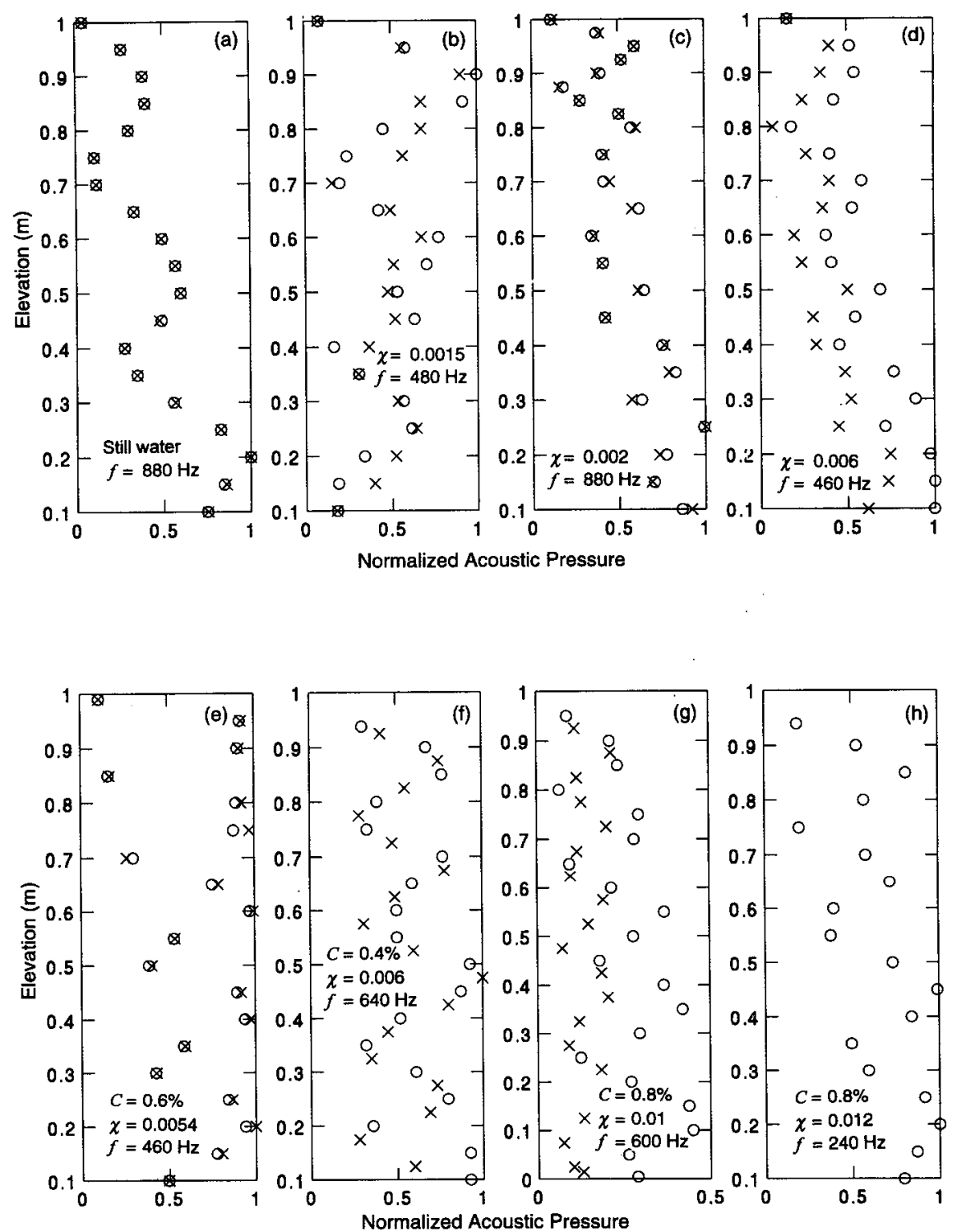

FlG. 8. The standing wave pattern of acoustic waves in bubbly water [top row, (a)-(d)] and artificial bubble clouds stabilized in a polymer mixture [bottom row, (e)-(h)]. The void fraction $x$, polymer concentration $C$, and acoustic frequency $f$ are specified on each panel.

range of void fractions used in the experiment was $0.0015-0.006$. The largest uncertainty on the determination of the void fraction, however, was the bubbly surface, which made the reading of the water surface elevation somewhat subjective. Because a complete measurement cycle typically lasts between 1 and $2 \mathrm{~h}$, four to five readings of the water surface elevations were taken for an average. No attempt was made to measure the bubble size distribution, but most of the bubbles were visually estimated to be smaller than approximately $2 \mathrm{~mm}$ at higher airflow rates. At lower flow rates, the bubble size could be controlled to be in the submillimeter range. (b) Acoustic measurements in polymer mixture: In this series, the tube was filled with an aerated polymer mixture. The void fraction and size distribution were determined by the methods described earlier. The range of void fractions was from 0.004 to 0.012 .

The acoustic frequencies used were from 240 to 880 $\mathrm{Hz}$, which are much lower than the resonance frequency of the constituent bubbles.

\section{2) RESULTS}

Examples of the standing wave pattern from this experiment are shown in Fig. 8. The four cases on the 

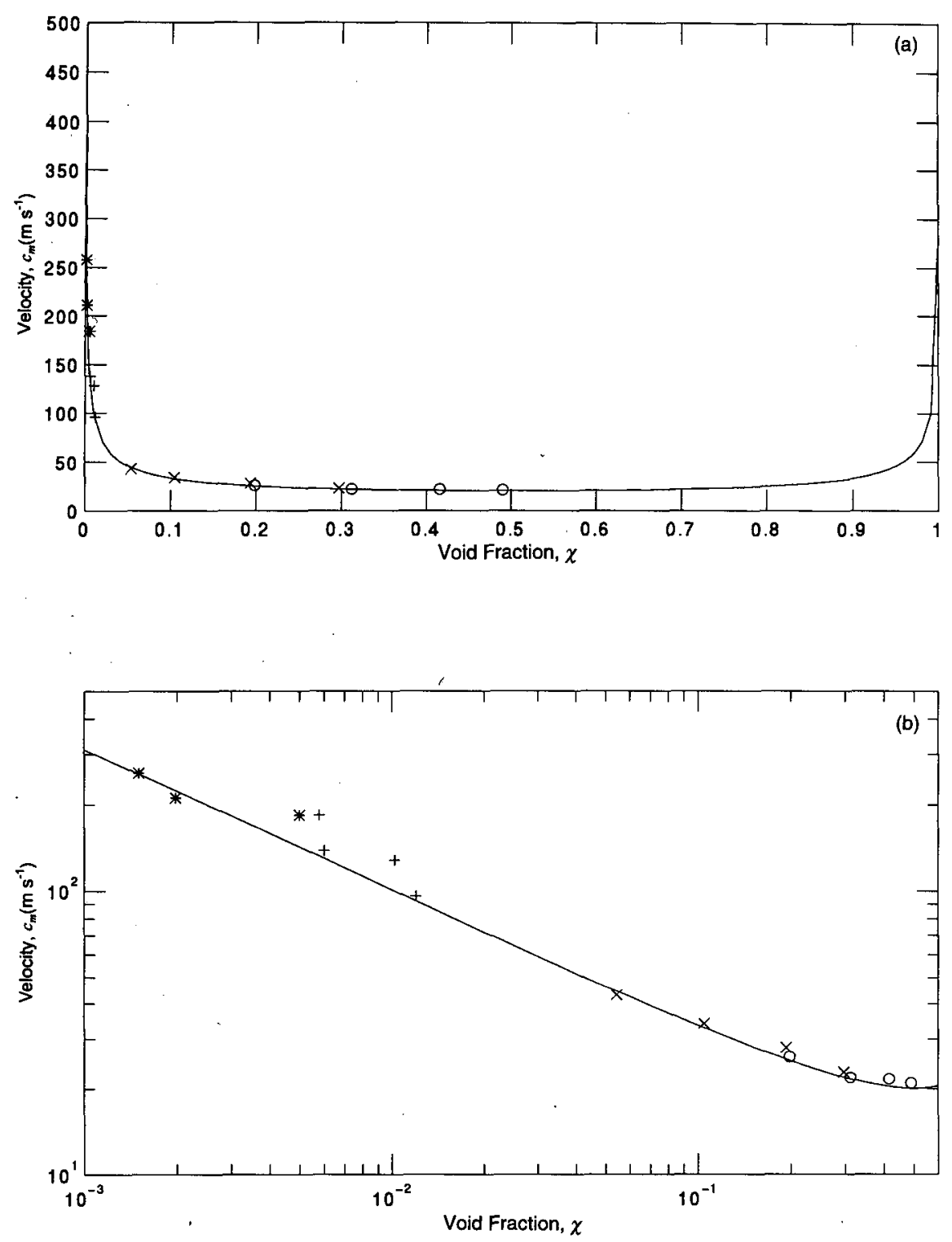

FIG. 9. The acoustic velocity in bubbly water and polymer mixture as derived from the resonant tube experiment. Symbols: asterisk - bubbly water, plus-polymer mixture, from present experiment; circle, cross-results from Karplus (1958), $f=1000$ and $500 \mathrm{~Hz}$, respectively; solid curvecalculations from Wood (1930). (a) Linear scale; (b) logarithmic scale.

top row show measurements taken in bubbly water. The void fraction and the acoustic frequency are labeled in each panel. The measurement repeatability is demonstrated by different symbols denoting the two traversing sequences mentioned in the procedure section. As a reference, the standing wave pattern in stationary water was also measured. The void fraction of this could not be determined from our experimental procedure, which depended on the elevation difference before and after bubble injection into the water column. The results indicate that the procedure of acoustic measurements is very repeatable. The void fractions for the other three bubbly water cases are $0.0015,0.002$, and 0.006 . The data scatter is considerably larger than that in the stationary water case. The signal fluctuations are partially attributed to the relatively inhomogeneous and unsteady bubble flow. Regardless, the standing wave pattern is clear for the wavelength measurement and acoustic velocity calculation using the procedure described earlier.

Four measurements in polymer mixtures are shown in the bottom row of Fig. 8. The polymer concentrations range from $0.4 \%$ to $0.8 \%$ and the void fractions from 0.004 to 0.012 (labeled in each plot). The repeatability of these measurements is substantially better than that for the bubbly water cases at an equivalent 

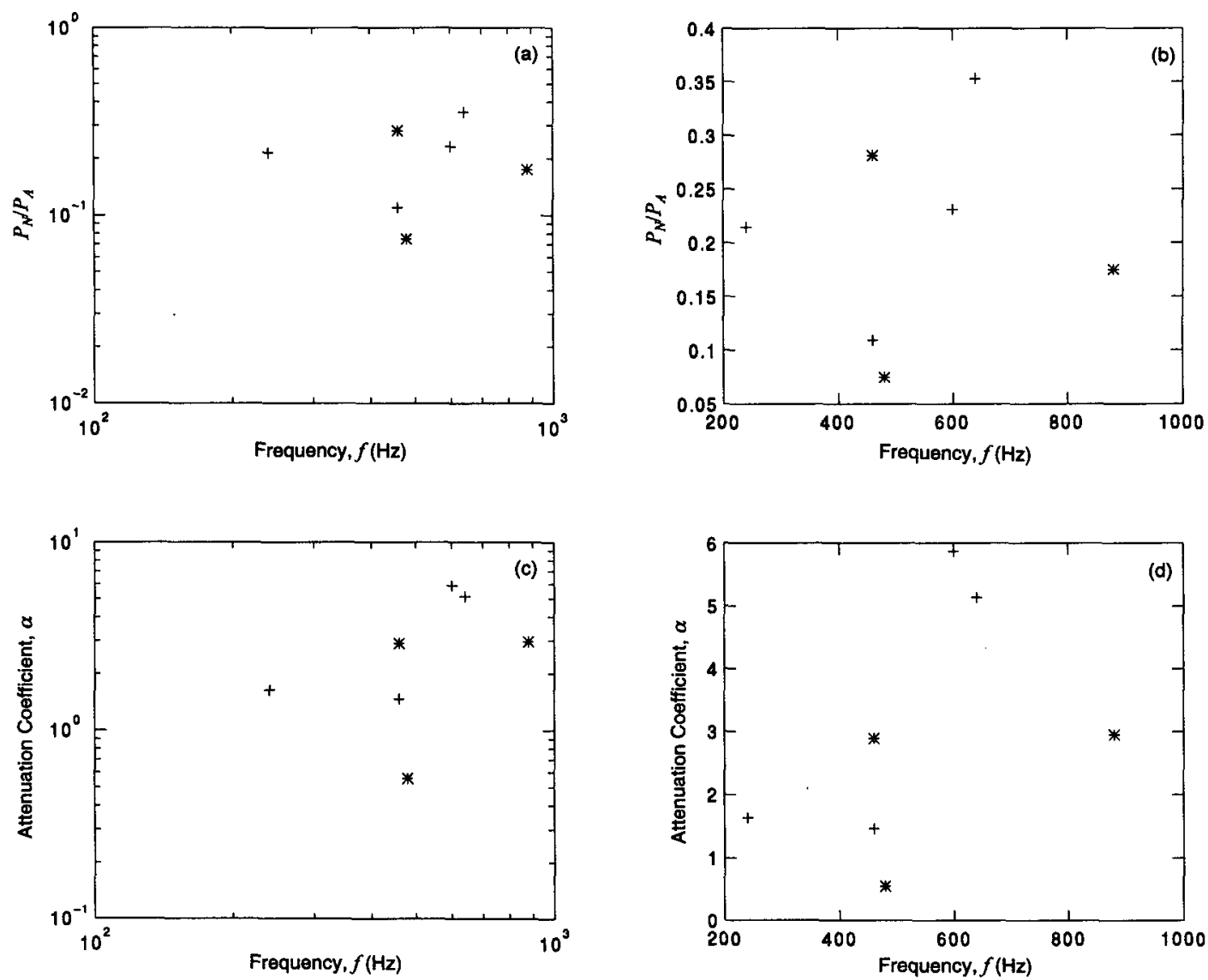

FIG. 10. The calculated $P_{N} / P_{A}(\mathrm{a}, \mathrm{b})$ and $\alpha(\mathrm{c}, \mathrm{d})$ from the resonant tube experiment. Symbols: asterisk—bubbly water; plus-polymer mixture. These data are presented in both logarithmic (left) and linear (right) scales.

void fraction. An artificial bubble cloud is, therefore, an attractive medium for the study of the acoustic properties of liquid containing air bubbles.

The acoustic velocity derived from these measurements is plotted in Fig. 9. For comparison, the theoretical results of Wood (1930) and experimental measurements of Karplus (1958) are also plotted on the figure. The agreement between data and theory is very good; the scatter of data is mainly contributed by the uncertainty of void fraction determination, as mentioned earlier. Temperature also affects the acoustic velocity in the medium; this factor was not taken into consideration in the data reduction.

An estimation of the attenuation coefficient was also attempted using the ratio of the acoustic pressure at the node and antinode of the standing wave pattern (Silberman 1957). The calculated results from this experiment were about one order of magnitude higher than those reported in Silberman (1957). This discrepancy was considered to be mainly caused by the large spacing used for mapping out the standing wave pattern: the spacing was either 0.025 or $0.05 \mathrm{~m}$, while the half-wavelength was approximately $0.15 \mathrm{~m}$ (Fig. 8 ). A more accurate procedure would have searched for the true positions of the nodes and antinodes to measure the acoustic pressure at the nodes and antinodes. Our effort to derive the quantitative magnitude of the absorption coefficient cannot be considered successful, and further improvement of the experimental technique is needed. Regardless of this lack of quantitative accuracy, results from the two experimental sequences shed light on the qualitative effects of polymer addition to acoustic absorption. The results of $P_{N} /$ $P_{A}$ and $\alpha$ are shown in Fig. 10. The scatter of data points is large for both sequences of the bubbly water and polymer mixture experiments. It is worthwhile to point out, though, that the measured attenuation coefficient of the polymer cases is not larger than the corresponding measurements in the bubbly water cases.

\section{Summary}

It is well known that the acoustic properties of a fluid, specifically the effective compressibility and the sound velocity, are greatly modified by a small amount of air bubbles in the liquid. The impedance of the aerated fluid is, therefore, very different from the ambient unaerated fluid. These characteristics are ideal for de- 
veloping bubble-based acoustic targets for underwater acoustics research and acoustic tracers for remote sensing of the ocean environment. Artificial bubble clouds can be produced by using polymer additives to suspend and stabilize air bubbles in the mixture. The stability of bubble clouds ranges from a few hours to a few months depending on the polymer concentration used. From resonant tube measurements, it is confirmed that the acoustic velocity in the polymer-suspended bubble mixture is also greatly reduced. The velocity reduction is comparable to that in bubbly water and is in agreement with the prediction using Wood's equation.

Experimental research of the acoustic properties of bubble clouds can be performed much more efficiently using stable artificial bubble clouds, as underwater bubbling usually produces unsteady bubble clouds. Because a very small amount of air bubbles and polymers (both less than $1 \%$ ) are needed to make an effective acoustic scatterer, the physical properties of the artificial bubble clouds can be easily adjusted to those of the ambient seawater; therefore, the target will follow the fluid motion truthfully. The artificial bubble clouds can also be shaped into any geometry to simulate various physical features, such as the bubble plumes or bubble clouds produced by wave breaking at different stages. Acoustic measurements of bubble cloud scattering can be made in the field or in the laboratory without the necessity to deal with wave breaking and the rough surface conditions associated with high sea states, which produce the wave breaking that generates the bubble plumes.

Acknowledgments. The research presented in this article was sponsored by ARPA under Contract DAAH01-93-C-R258. Jack Kolle contributed to the concept of using a polymer solution to suspend air bubbles and supplied Fig. 3; Dan Heiss performed most of the experiments and made many careful observations; and Jeff Schindall provided the computation for Fig. 2c. We also acknowledge the constructive comments and suggestions from two anonymous reviewers; the comments from one reviewer contributed to the comparison study of different formulas for the bubble cloud resonance frequency presented in the appendix. The completion of this paper is also supported by QUEST Integrated, Inc., under IR\&D funding.

\section{APPENDIX}

\section{Approximate Equations of Bubble Cloud Resonance Frequency}

The study of the acoustic and hydrodynamic properties of bubble clouds and mixtures of liquid and gas bubbles is quite extensive. A small collection of the literature, by no means exhaustive, is cited in the references. These research results contribute to the formulation of the idea of using artificial bubble clouds for underwater acoustics research and ocean rennote sensing. A detailed review of the literature is beyond the scope of this paper. The results from these studies are not fully compatible. One example is the calculation of the resonance frequency of a bubble cloud, in which a numerical difference by a factor of 2 or more may exist. In this appendix, the results of four of the commonly used equations are compared. In the following presentation, $f$ represents the resonance frequency of a bubble cloud, and the subscripts 1,2,3, and 4 denote the different sources of these equations. The definitions of the other symbols are identical to those used in the text.

The first equation is introduced by Carey and his coworkers (Carey and Roy 1993; Carey and Fitzgerald 1993) using a Green's function approach to solve the boundary value problem:

$$
f_{1}=\frac{1}{2 \pi R}\left(\frac{3 \gamma P_{A}}{\rho \chi}\right)^{1 / 2} .
$$

A detailed discussion of the theoretical derivation is given in Carey and Roy. They emphasize that their results are found in the classical work of Anderson (1950), Rschevkin (1963), and Morse and Ingard (1968). The solutions obtained yield the same expressions for resonance frequency and backscatter cross section when Wood's approximation is used. This expression is used in our calculations presented in the main text.

The following equation, introduced by Omta (1987), is derived from solving the linearized equation of motion of a bubble cloud surrounded by fluid extending to infinity:

$$
f_{2}=\frac{1}{4 R}\left(\frac{\gamma P_{A}}{\rho \chi}\right)^{1 / 2} .
$$

Following the same solution procedure, slightly different equations are given in d'Agostino and Brennen (1988):

$$
f_{3}=\frac{1}{4 R}\left\{\frac{3 \gamma P_{A}}{\rho\left[3 \chi+(\pi a / 2 R)^{2}\right]}\right\}^{1 / 2}
$$

and in Prosperetti et al. (1993):

$$
f_{4}=\frac{1}{4 R}\left\{\frac{3 \gamma P_{A}}{\rho\left[3 \chi(1-\chi)+(\pi a / 2 R)^{2}\right]}\right\}^{1 / 2}
$$

A few differences among these expressions can be readily observed: (a) There is a constant ratio of $\pi(2 \sqrt{3})^{-1}=0.91$ between $f_{1}$ and $f_{2}$; that is, (A2) predicts a resonance frequency about $9 \%$ lower than (A1). (b) The predictions of $f_{3}$ and $f_{4}$ approach each other as the void fraction decreases; the resonance frequency predicted by (A3) is higher than that from (A4) by a factor of approximately $(1-\chi)^{1 / 2}$. (c) Equations (A1) and (A2) indicate that the resonance frequency of a bubble cloud is independent of the 
Bubble Size: $0.1 \mathrm{~mm}$
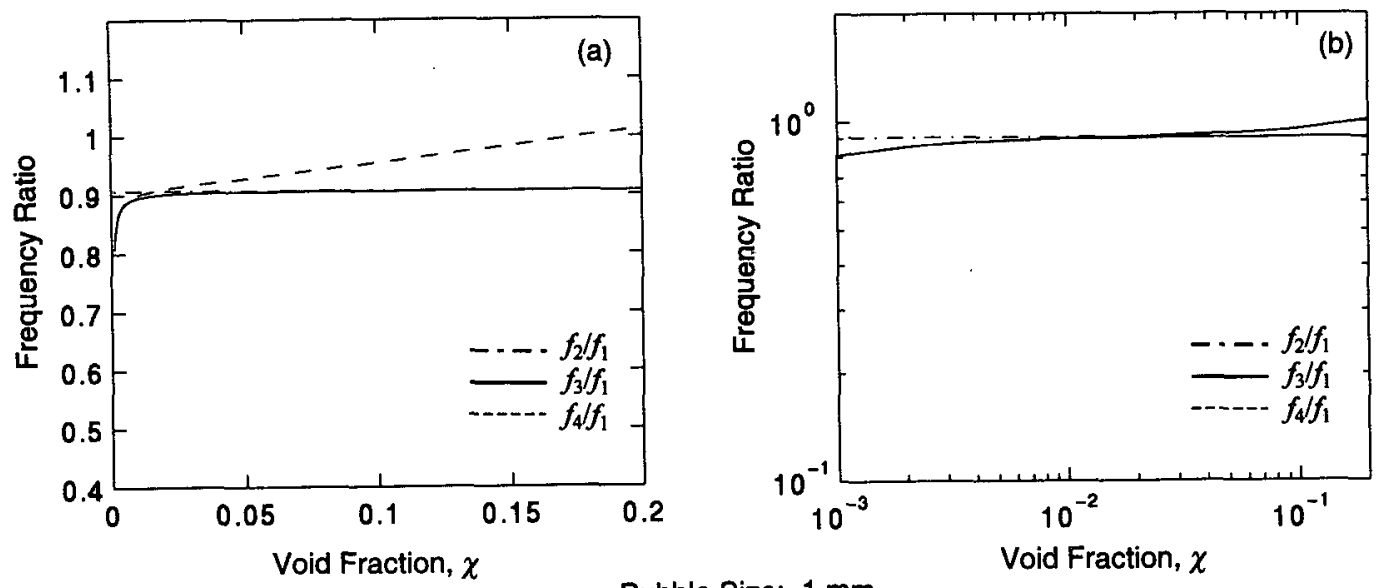

Bubble Size: $1 \mathrm{~mm}$
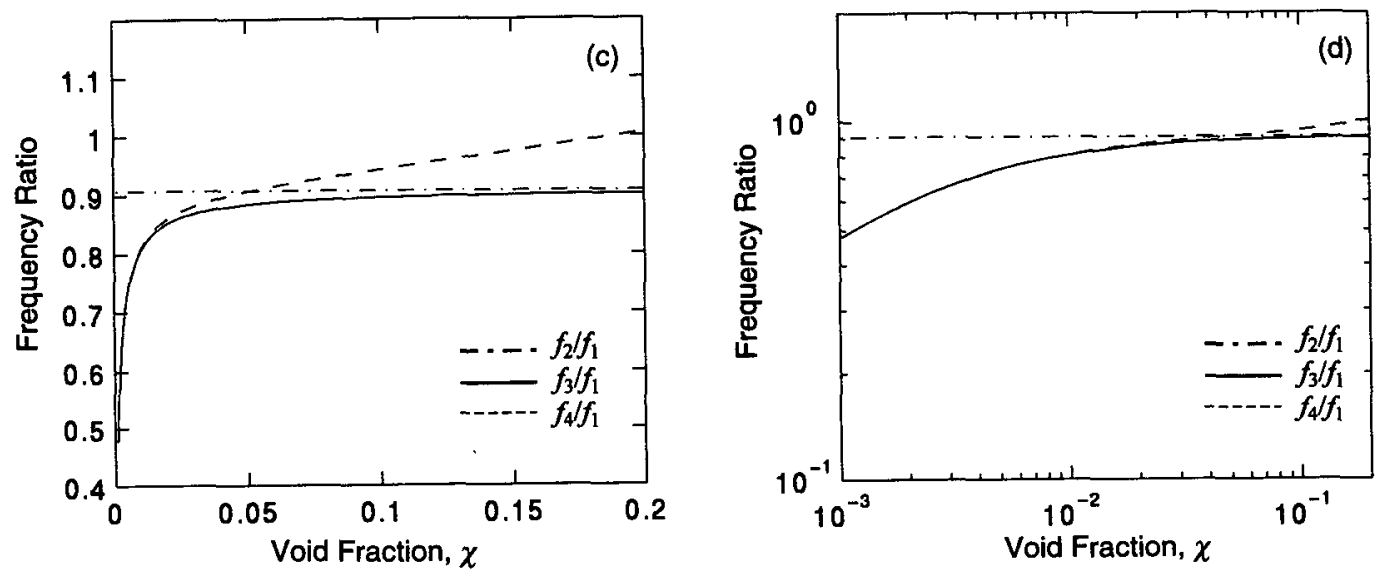

Bubble Size: $3 \mathrm{~mm}$
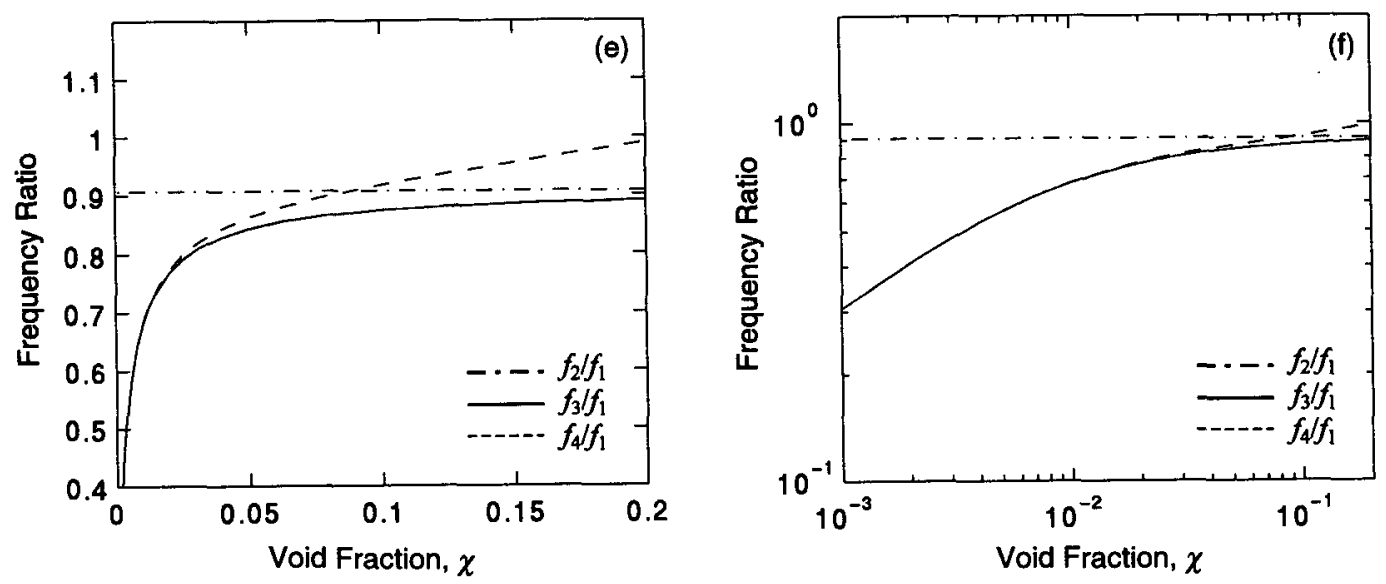

FIG. A1. The ratio of the bubble cloud resonance frequency calculated from four different equations [(A1)-(A4)]. Dashed-and-dotted: $f_{2} / f_{1}$; solid: $f_{3} / f_{1}$; dashed: $f_{4} / f_{1}$. The bubble size required for the application of (A3) and (A4) is (a, b) $0.1 \mathrm{~mm}$; (c, d) $1 \mathrm{~mm}$; and (e, f) $3 \mathrm{~mm}$. The ratio is plotted in both linear (left) and logarithmic (right) scales.

size of the constituent bubbles. In contrast, the size dependence in (A3) and (A4) is explicit; the significance of the size dependence increases toward a low void fraction.
As an illustrative example, Fig. A1 shows the ratios of $f_{2} / f_{1}$ (dashed-and-dotted curve), $f_{3} / f_{1}$ (solid curve), and $f_{4} / f_{1}$ (dashed curve) calculated for the range of void fractions from 0.001 to 0.20 . The radius of the 
bubble cloud is $0.2 \mathrm{~m}$. In general, the last three formulas predict lower resonance frequencies than the first one. All four equations agree with each other within $10 \%$ except at low void fractions. For example, at a 0.001 void fraction, the ratios of $f_{3} / f_{1}$ and $f_{4} / f_{1}$ are $0.8,0.47$, and 0.30 with the radii of constituent bubbles $0.1,1$, and $3 \mathrm{~mm}$, respectively.

The question of the dependence of bubble size on bubble cloud resonance has not been resolved, either through theoretical arguments or from experimental evidence. It is not easy to perform experiments with bubble clouds even in the laboratory, mainly because of the unstable nature of the generated bubble plume and the fact that low-frequency radiation requires large facilities. The first problem can be solved by using stable artificial bubble clouds in the experiments.

\section{REFERENCES}

Anderson, V., 1950: Sound scattering from a fluid sphere. J. Acoust. Soc. Amer., 22, 426-431.

Blanchard, D. C., 1983: The production, distribution, and bacterial enrichment of the sea-salt aerosol. Air-Sea Exchange of Gases and Particles, P. S. Liss and W. G. N. Slinn, Eds., D. Reidel Publishing, 407-454.

- and L. D. Syzdek, 1972: Concentration of bacteria in jet drops from bursting bubbles. J. Geophys. Res., 77, 5087-5099.

Carey, W. M., 1985: Low-frequency ocean surface noise sources. $J$. Acoust. Soc. Amer., 78(Suppl. 1), S1-S2.

- 1 1987: Low frequency noise and bubble plume oscillations. $J$. Acoust. Soc. Amer. 82(Suppl. 1), $\$ 62$.

- , and D. G. Browning, 1988: Low-frequency ocean ambient noise: Measurements and theory. Sea Surface Sound, B. R. Kerman, Ed., Kluwer Academic, 361 376.

- , and R. A. Roy, 1993: Sound scattering from microbubble distributions near the sea surface. Ocean Reverberation, D. D. Ellis, J. R. Preston, and H. G. Urban, Eds., Kluwer Academic 2543.

_ and J. W. Fitzgerald, 1993: Low-frequency noise from breaking waves. Natural Physical Sources of Underwater Sound, B. R. Kerman, Ed., Kluwer Academic, 277-304.

- — - E. C. Monahan, and Q. Wang, 1993: Measurement of sound produced by a tipping trough with fresh and salt water. J. Acoust. Soc. Amer., 93, 3178-3192.

Carsten, E. L., and L. L. Foldy, 1947: Propagation of sound through a liquid containing bubbles. J. Acoust. Soc. Amer., 19, $481-$ 501.

Clay, C. S., and H. Medwin, 1977: Acoustical Oceanography: Principles and Applications. Wiley-Interscience Publishing, 544 pp.

Commander, K. W., and A. Prosperetti, 1989: Linear pressure waves in bubbly liquids: Comparison between theory and experiments. J. Acoust. Soc. Amer., 85, 732-746.

Coste, C., and C. Laroche, 1993: Acoustic behavior of a liquid/vapour mixture in a standing-wave tube. J. Fluid Mech., 246, 67-89.

Crum, L. A., J. A. Schindall, R. A. Roy, and W. M. Carey, 1994: Low frequency resonance backscatter from near-surface bubble clouds. Proc. NATO Low-Frequency Active Sonar Symposium, Kluwer Academic, in press.

d'Agostino, L., and C. E. Brennen, 1988: Acoustical absorption and. scattering cross sections of spherical bubble clouds. $J$. Acoust. Soc. Amer., 84, 2126-2134.

- , and,- 1989 : Linearized dynamics of spherical bubble clouds. J. Fluid Mech., 199, 155-176.

Devin, C., 1959: Survey of thermal, radiation and viscous damping of pulsating air bubbles in water. J. Acoust. Soc. Amer., 31, 1654-1667.
Gray, G. R., and H. C. H. Darley, 1981: Composition and Properties of Oil Well Drilling Fluids. 4th ed. Gulf Publishing Co., 630 $\mathrm{pp}$.

Hwang, P. A., R. A. Roy, and L. A. Crum, 1995: Artificial. bubble cloud targets. Sea Surface Sound'94: Third International Meeting on Natural Physical Processes Related to Sea Surface: Sound, M. J. Buckingham and J. R. Porter, Eds., World Scientific Publ., in press.

Karplus, H. B., 1958: The velocity of sound in a liquid containing gas bubbles, Armour Res. Found., ITT Res. U.S. Atomic Energy Commission, NTIS COO-248, $40 \mathrm{pp}$.

Kelco, 1988: Xanthan gum-Natural biogum for scientifıc water control. Tech. booklet, $3 \mathrm{~d}$ ed. Merck \& Co., Inc., $24 \mathrm{pp}$.

Kerman, B. R., 1984: Underwater sound generation by breaking wind waves. J. Acoust. Soc. Amer., 75, 149-165.

- - (ed.), 1988: Sea Surface Sound: Natural Mechanisms of Surface Generated Noise in the Ocean. Kluwer Academic, $639 \mathrm{pp}$.

- - Ed., 1993: Natural Physical Sources of Underwater Sound. Kluwer Academic, 750 pp.

Kolaini, A., R. A. Roy, and L. A. Crum, 1991: An investigation of the acoustic emissions from a bubble plume. J. Acoust. Soc. Amer., 89, 2452-2455.

Kumar, S., and C. E. Brennen, 1991: Nonlinear effects in the dynamics of clouds of bubbles. J. Acoust. Soc. Amer., 89, 707-714.

$\longrightarrow$, and - , 1993: Some nonlinear interactive effects in bubbly clouds. J. Fluid Mech., 253, 565-591.

Loewen, M. R., and W. K. Melville, 1994: An experimental investigation of the collective oscillations of bubble plumes entrained by breaking waves. J. Acoust. Soc. Amer., 95, 1329-1343.

Lu, N. Q., A. Prosperetti, and S. W. Yoon, 1990: Underwater noise emission from bubble clouds. IEEE J. Ocean. Eng., 15, 275281.

Minnaert, M., 1933: On musical air-bubbles and the sounds of running water. Phil. Mag., 16, 235-248.

Morse, P. M., and K. Ingard, 1968: Theoretical Acoustics. McGrawHill Book Co., 927 pp.

Omta, R., 1987: Oscillation of a cloud of bubbles of small and not so small amplitude. J. Acoust. Soc. Amer., 82, 1018-1033.

Prosperetti, A., 1985: Bubble-related ambient noise in the ocean. $J$. Acoust. Soc. Amer., 78(Suppl. 1), S2.

- 1988 a: Bubble-related ambient noise in the ocean. J. Acoust Soc. Amer., 84, 1042-1054.

_- 1988b: Bubble dynamics in ocean ambient noise. Sea Surface Sound, B. R. Kerman, Ed., Kluwer Academic, 151-171.

- N. Q. Lu, and H. S. Kim, 1993: Active and passive acoustic behavior of bubble clouds at the ocean's surface. J. Acoust. Soc. Amer., 93, 3117-3127.

Roy, R. A., W. Carey, M. Nicholas, J. Schindall, and L. A. Crum, 1992: Low-frequency scattering from submerged bubble clouds. J. Acoust. Soc. Amer., 92, 2993-2996.

—, J. A. Schindall, W. M. Carey, and L. A. Crum, 1994: Can near-surface bubble clouds and plumes lead to anomalous perturbations in low-frequency sea-surface scattering? Proc. Second European Congress on Underwater Acoustics, Luxembourg, European Commission, Luxembourg, 195-202.

Rschevkin, S. N., 1963: The Theory of Sound. Pergamon Press, 464 pp.

Schindall, J., W. M. Carey, R. A. Roy, L. A. Crum, and M. Nicholas, 1992: Sound scattering from near-surface bubble clouds. $J$. Acoust. Soc. Amer., 92, 2392.

Silberman, E., 1957: Sound velocity and attenuation in bubbly mix tures measured in standing wave tubes. J. Acoust. Soc. Amer., 29, 925-933.

Urick, R. J., 1967: Principles of Underwater Sound for Engineers. McGraw-Hill, 342 pp.

Ward, C. A., P. Tikuisis, and R. D. Venter, 1982: Stability of bubbles in a closed volume of liquid-gas solution. J. Appl. Phys., 53, 6076-6080.

Wood, A. B., 1930: A Textbook of Sound. Macmillan, $568 \mathrm{pp}$.

Yoon, S. W., L. A. Crum, A. Prosperetti, and N. Q. Lu, 1991: An investigation of the collective oscillations of a bubble cloud. $J$. Acoust. Soc. Amer., 89, 700-706. 